\title{
Design, construction and test of a lightweight Thomson Coil actuator for medium voltage vacuum switch operation
}

DOI:

10.1109/TEC.2019.2905745

\section{Document Version}

Accepted author manuscript

Link to publication record in Manchester Research Explorer

\section{Citation for published version (APA):}

Vilchis-Rodriguez, D., Shuttleworth, R., Smith, A. C., \& Barnes, M. (2019). Design, construction and test of a lightweight Thomson Coil actuator for medium voltage vacuum switch operation. IEEE Transactions on Energy Conversion, 0-11. [TEC-00966-2018]. https://doi.org/10.1109/TEC.2019.2905745

\section{Published in:}

IEEE Transactions on Energy Conversion

\section{Citing this paper}

Please note that where the full-text provided on Manchester Research Explorer is the Author Accepted Manuscript or Proof version this may differ from the final Published version. If citing, it is advised that you check and use the publisher's definitive version.

\section{General rights}

Copyright and moral rights for the publications made accessible in the Research Explorer are retained by the authors and/or other copyright owners and it is a condition of accessing publications that users recognise and abide by the legal requirements associated with these rights.

\section{Takedown policy}

If you believe that this document breaches copyright please refer to the University of Manchester's Takedown Procedures [http://man.ac.uk/04Y6Bo] or contact uml.scholarlycommunications@manchester.ac.uk providing relevant details, so we can investigate your claim.

\section{OPEN ACCESS}




\title{
Design, construction and test of a lightweight Thomson Coil actuator for medium voltage vacuum switch operation
}

\author{
D.S. Vilchis-Rodriguez, Member, IEEE, R. Shuttleworth, A.C. Smith, Member, IEEE \\ and M. Barnes, Member, IEEE
}

\begin{abstract}
This paper details the design, construction and test of a lightweight, low energy usage Thomson Coil actuator for the operation of a commercial medium voltage vacuum switch. A novel latching design, directly linked to the actuator armature, helps to keep the actuator mass at minimum. Experimental results show that $125 \mathrm{~J}$ of electric energy are sufficient for operation of a commercial $27 \mathrm{kV}$ vacuum interrupter in times suitable for medium voltage DC protection applications. The use of high conductor density coils is shown to play a key role on the observed actuator performance. Finite element simulations are used to demonstrate that shorter operation times are feasible, without augmenting the consumed electrical energy, by further increasing coil conductor density.
\end{abstract}

Index Terms - DC breaker, magnetic repulsion, Thomson Coil, ultra-fast actuator, vacuum switch.

\section{INTRODUCTION}

$\mathrm{M}$ EDIUM Voltage DC (MVDC) networks are being actively researched for use in future distribution systems, such as all-electric shipboard power systems [1-3], aircraft propulsion systems [4], micro grids [5, 6] and wind farm collector systems [7]. Multi-terminal, meshed grids may significantly enhance the stability, reliability and security of operation of DC networks. Due to their flexibility of operation, voltage source converter (VSC) technologies are preferred on these networks. For economic, safe and continuous operation of VSC based DC networks the development of fast acting, low loss DC breakers is essential [8]. For instance, once a DC fault is detected, a breaker isolation time of about $2 \mathrm{~ms}$ is suggested as necessary to preserve network integrity in HVDC grids [8]. For MVDC network protection similar breaking times have been targeted [9]. Hybrid DC breaker designs are able to fulfil the stringent requirements demanded for $\mathrm{DC}$ protection in the medium and high voltage range [10-12], although the advent of wide band gap (WBG) power devices is starting to make the use of solid state DC breakers competitive in MV applications [13]. A

This work was funded as part of the UK EPSRC, FCL/B: An Integrated VSC-HVDC Fault Current Limiter/Breaker project, EP/L021552/1.

D.S. Vilchis-Rodriguez, R. Shuttleworth, A.C. Smith and M. Barnes are with the Power \& Energy Division, School of Electrical and Electronic Engineering, The University of Manchester, Manchester M13 9PL, UK. (email: Damian.Vilchis-Rodriguez@ manchester.ac.uk). hybrid DC breaker combines solid state components and an ultra-fast mechanical switch to meet the low on state losses and short breaking time required for practical DC protection applications [10-12]. The mechanical switch on a hybrid DC breaker offers a low impedance conduction path under normal operation, while providing a galvanic barrier under fault conditions. Owing to the time scale differences between electrical and mechanical systems, the opening speed of the mechanical switch has a decisive impact on the breaker isolation time. Unsurprisingly, a large portion of a hybrid DC breaker operation time is spent on mechanical switch opening [10-12]. Therefore the use of an ultra-fast actuator, paired with a robust mechanical switch is necessary to expedite and warrant effective operation of hybrid DC breakers.

The Thomson Coil (TC) actuator is a simple, robust device capable of ultra-high speed operation that works under the principle of magnetic (eddy current) repulsion. Its robustness and high speed capabilities make the TC actuator highly suitable for applications where low maintenance and high performance are demanded, such as in DC breakers, current limiters and load commutation switches [9-12, 14-18]. However, in spite of its many operational advantages, the TC actuator exhibits an important drawback: poor electrical to mechanical energy conversion efficiency, typically about $5 \%$ $[19,20]$. Thus TCs tend to use comparatively more energy than competing schemes for similar displacements and operating times [20]. Consequently, a relatively large amount of energy must be used to achieve the required operating time, impacting negatively on size and rating of the actuator ancillary devices. One recommended approach to improve actuator efficiency, and therefore reduce energy consumption and operation time, is actuator moving mass minimization [19, 20]. Thus a low mass switching device must be ideally paired to a similarly light TC design to improve breaker performance. From the existing switching technologies the Vacuum Interrupter (VI) exhibits many characteristics that make it highly suitable for MV protection applications. Characteristics such as compact design, maintenance free operation, long service life, short stroke requirement, low energy demands and environmental neutrality have made the VI the dominant switching technology in MV applications. In addition, due to environmental concerns, VI usage is slowly extending towards high voltage applications, where $\mathrm{SF}_{6}$ is the predominant 
switching technology today. Due to its many advantages it is unsurprising that the pairing of a TC and a VI has been considered in multiple publications $[9,11,13,16,23-30]$. However the actuator energy requirements under load have been often ignored, overshadowed by the apparatus speed requirements. In this paper the operation of a TC-VI pair is investigated. A lightweight $\mathrm{TC}$ actuator is designed and constructed to be paired with a commercial $27 \mathrm{kV}$ VI. An operation time of $2 \mathrm{~ms}$ is verified and the TC-VI pair energy requirements are quantified. Design choices are explained and further improvements to the design explored with the help of Finite Element Analysis (FEA) simulations.

\section{ACTUATOR DESIGN}

The TC actuator typically consists of a metallic armature, two spiral coils (one for opening and one for closure), a transmission/insulation rod to mechanically link the actuated device (i.e. VI), a latching mechanism, a damping system, electric energy storage and control ancillaries. To initiate displacement, one of the actuator's coils is excited by a time varying current, usually produced by capacitor discharge, creating a time varying magnetic field that interacts with the metallic armature, inducing eddy currents within it. Due to the direction of the induced eddy currents, a repulsive magnetic force appears between coil and armature, enabling rapid armature reaction and high speed displacement. The latching mechanism is designed to keep the switch in a stable open position and provide adequate contact force in the closed position. The damping system meanwhile is responsible for the soft stop of the armature travel, to avoid mechanical damage and therefore accelerated aging of the mechanical components. The total moving mass of the TC-VI pair is comprised by the mass of the actuator moveable components and the VI moveable electrode. The TC moving parts usually include the actuator's armature, transmission rod and the auxiliary components required to physically connect damping and latching systems. Such auxiliary components are normally attached to the transmission bar [23-30]. For soft stop of the armature, passive and active damping systems have been proposed [29-33]. Depending on the selected damping principle, mass may or may not be added to the system moveable mass. For instance, hydraulic systems like that described in [25] require the extension and reinforcement of the transmission rod to physically interface the damper and the moveable elements. Magnetic dampers like the one discussed in [32] require PM and ferromagnetic material to be attached to the moving components to complete the necessary magnetic circuit, thus increasing moveable mass. On the other hand, the use of active and passive damping mechanisms such as those proposed in [29] and [33], respectively, may be used to avoid increased moveable mass. For versatility and simplicity of implementation the active damping scheme proposed in [29] is considered in this work.

\section{A. Latch design}

For TC latching, magnetic $[24,28]$ and spring loaded bistable mechanisms have been preferred [25-27]. However these systems tend to increase the moving mass, due to the appendages needed to mechanically or magnetically couple latching and actuator systems, Fig. 1. In this paper the use of a bistable mechanism, based on the buckling phenomenon [34, 35], directly attached to the armature body is proposed. The suggested arrangement adds negligible mass to the system compared to other designs, while providing adequate latching force. Fig. 2 shows a schematic representation of the proposed implementation. As can be seen in the figures, the proposed design eliminates the need for any rod extension, appendage or clamping component to mechanically link armature and latching systems, thus reducing the number of system components, moveable mass and simplifying the mechanical design.

To design the latching system, stationary 3D Finite Element (3D-FE) simulations were conducted using the solid mechanics interface of the COMSOL 5.3 multi-physics software. Geometric nonlinearities were considered in the model solution to account for the deformation of the latching ribbons. Thus in the calculations the strains and stresses are represented by the Green-Lagrange strain and the Second Piola-Kirchoff stress tensors, respectively. The buckling system is assumed to be made of $1 \mathrm{~mm}$ thick, $10 \mathrm{~mm}$ wide, $8.5 \mathrm{~cm}$ long CK75 spring steel ribbons. Spring steels are steel alloys that can achieve very high yield strength close to the material tensile strength [36-38]. For reference, quenched and tempered (+QT) CK75 spring steel alloys' tensile strength values are in the range of 1200-1900 MPa.
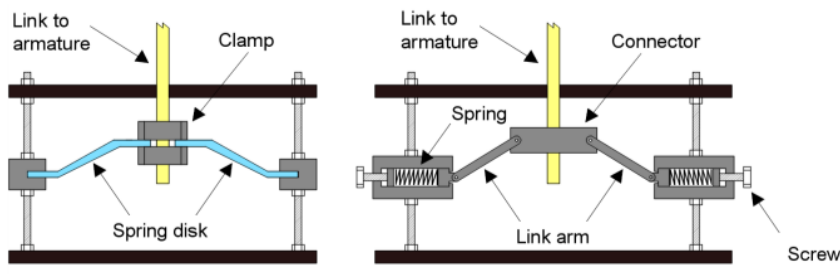

Fig. 1. Spring loaded latching mechanisms proposed in [26] (left) and [27] (right) for use with a TC actuator.

(a)

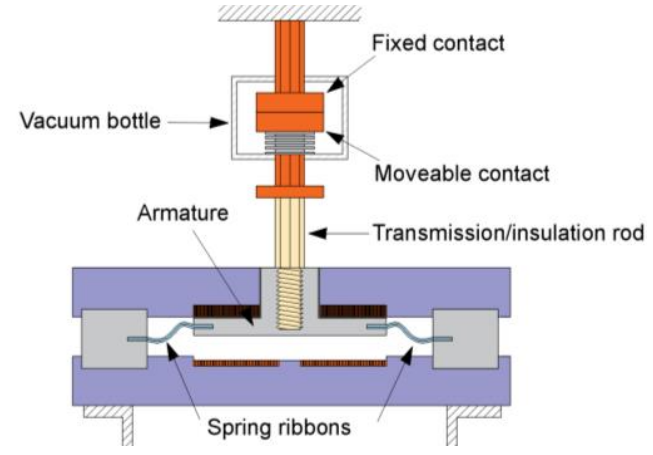

(b)

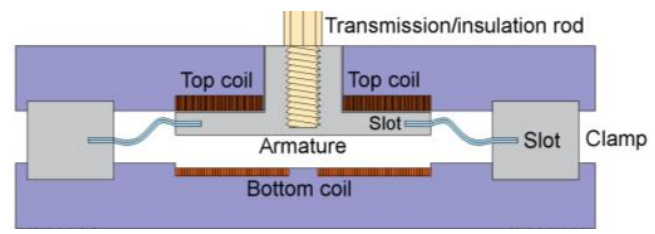

Fig. 2. (a) Cross section diagram of proposed VI-TC actuator design and (b) latching mechanism detail. 
Due to its relatively high conductivity to weight ratio, aluminum alloy was chosen as the armature material. A 6082T6 aluminum armature, with an outer diameter of $10 \mathrm{~cm}$ and a thickness of $9 \mathrm{~mm}$ is considered in the analysis. The rationale behind the selected armature thickness is explained in more detail in section III. In the FE simulations, the armature displacement is restricted to the vertical direction. In the design, the ribbon ends are inserted into $1 \mathrm{~cm}$ deep slots, machined into the armature body and lateral clamps, see Fig. $2 \mathrm{~b}$. The separation between the armature external edge and the clamp internal edge is $6.4 \mathrm{~mm}$, thus the steel ribbons may be subject to a theoretical maximum compressive strain of 1/85.

Fig. 3 shows a result obtained from the stationary 3D-FE simulations illustrating the mechanism geometry. To obtain the F-d curve of the latching system it is assumed at first that the spring ribbons are subject to maximum compressive strain, with the armature standing at the zero position; at zero displacement the centers of the armature and clamps slots are aligned. Then the armature is moved to its uppermost location (i.e. half the stroke length) and posteriorly displaced in small steps toward its lowest position (i.e. minus half the stroke). The steady state solution in each intermediate position was found and the latching force calculated from the vertical component of the armature reaction force. Fig. 4 shows the Fd profile obtained from the FE simulation for an armature displacement of $\pm 7.1 \mathrm{~mm}$. As seen in Fig. 4, the latching mechanism produces a linear characteristic for the displacement range considered, providing maximum force at extreme positions. The peak latching force produced by this arrangement can be modified, for instance, by varying the ribbon strain or its dimensions. However care should be taken not to exert excessive stress in the ribbons, otherwise fracture or plastic deformation of the material may occur if its yield strength is exceeded. On the other hand, if the ribbon strain is too low, the operating range of the latching system can be shorter than the required stroke length. Thus the ribbon needs to be stretched beyond its original length (tensile strain) to allow the armature displacement over the required distance. This is illustrated in Fig. 5, where the F-d characteristic for the latching system is shown for different initial compressive strains. It should be noted that the shape of the F-d curves shown in Fig. 5 is essentially identical to that of bistable buckled beam mechanisms $[34,35]$. As can be seen in the figure, as the initial compressive strain on the ribbon is decreased, the linear portion of the F-d characteristic is reduced (i.e. the ribbon strain changes from compressive to tensile for shorter displacement), thus the usable range of the latching system decreases as well. Therefore, the geometric and mechanical properties of the material must be carefully considered to attain effective and long term operation of the latching system. It should be noted that although spring steel CK75 is assumed here, customized spring steels with yield strengths considerably higher than that of CK75 are available. Thus by using a different material, varying the ribbon strain, its geometry or physical dimensions (thickness, width or length) the latching system can be customized for the specific application.

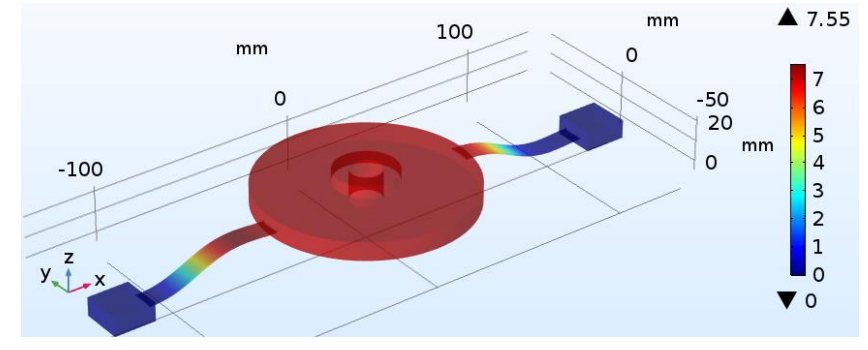

Fig. 3. TC latching mechanism displacement, stationary 3D-FE simulation.

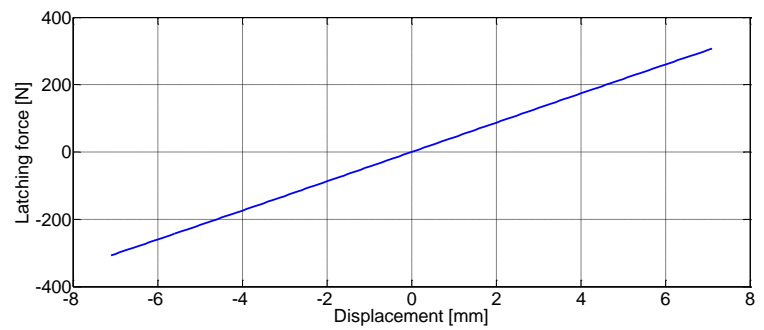

Fig. 4. Latching system F-d curve obtained from FE simulations.

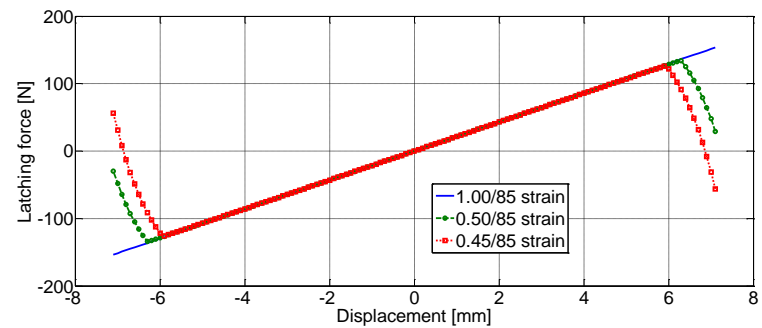

Fig. 5. F-d curve obtained from FE simulations for several compressive strains for a single latching ribbon.

\section{B. Actuator design performance analysis}

From the existing modelling tools, transient 2D FEA has been shown to provide accurate results for TC performance analysis [39]. Thus transient FE simulations are used here to assess the actuator operation under dynamic conditions, select the most convenient coil geometry and establish actuator energy requirements. A 2D axis-symmetric FE model was implemented using COMSOL 5.3 multi-physics software. COMSOL magnetic fields, solid mechanics and electrical circuit interfaces are used in the model implementation. In the multi-physics model, a lumped representation of the excitation circuit was interfaced with a FE representation of the coil centered in a large air domain. Then FE and electric circuit equations were solved simultaneously using a time dependent, fully coupled solver with an absolute tolerance of $10^{-3}$. Skin and proximity effects are also considered in the simulations. Fig. 6 shows a schematic representation of the electric circuit FE model interface; the same electric circuit is used for top and bottom coil excitation. Fig. 7 shows the geometry considered in the FE simulations, including transmission rod. The armature's neck was added to facilitate strong coupling between transmission rod and armature.

The armature mechanical behavior was modeled using COMSOL's solid mechanics interface, where isotropic linear elastic materials are assumed. The electromagnetic force acting over the armature was calculated within the mechanics interface as the combination of Lorentz force contribution and gravity effects. Vacuum and latching forces were added to the simulations as position dependent boundary forces acting on 
the vertical direction, over the top of the transmission rod and the armature outer edge, respectively. In this paper the use of a commercial VI, Jennings RP-127 [40], is considered during simulations and experimental tests. The VI relevant parameters are listed in Table I.

To implement a realistic representation of the force exerted by the vacuum switch in the simulations, the force required to keep the VI open at rated stroke length was measured by a static load test $(100 \mathrm{~N})$; then a linear distribution of vacuum force over the length of the electrode gap was adopted in the FE model. A deformable moving mesh was used to accurately account for armature displacement in the simulations and any aerodynamic effect was neglected. The equations used by the FEA software for the model solution are the same as in [39].

Since many capacitance and voltage combinations can be used for coil excitation, realistic restrictions were put in place in order to estimate the most convenient coil geometry. A capacitance-voltage combination of $1500 \mu \mathrm{F}-425 \mathrm{~V}$, compatible with existing laboratory equipment, was used to limit the electric energy available in the simulations for actuator operation to $135 \mathrm{~J}$. To operate the actuator, the thyristor switch (see Fig. 6) is closed to excite the top coil and initiate armature displacement. The thyristor conducts until the current falls below its holding current. As the armature moves downward, and the contact gap increases, the magnitude of the upward force exerted by the VI also increases. At the same time the latching force magnitude decreases, until it transitions from a positive (upward) to a negative (downward) direction, once the armature crosses the mid stroke. The negatively acting latching force helps to keep the armature moving in the downward direction, and holds the switch contacts in the open position once the end of travel has been reached. Active damping is provided by applying timely excitation to the bottom coil as the armature approaches the end of travel.

The proposed armature design, Fig. 2, eliminates the need to extend the transmission rod beyond the armature's lowest plane, leaving an untouched surface that can be used for example to fit the most convenient coil size. For instance, a coil with a higher conductor density usually increases armature reaction time but also improves actuator efficiency. A coil with such characteristics would be better suited for switch closing operation. In contrast, given that switch opening time is critical in DC breaker applications, a coil that allows the largest armature displacement for a given time and electric energy would be better suited for switch opening. Thus the larger flat surface existing at the bottom of the armature facilitates the installation of a larger coil, more suitable for switch closure.

To estimate the coil's most convenient conductor crosssection, parametric FE studies were conducted. First the conductor cross section height was kept constant at $6 \mathrm{~mm}$, while its thickness was varied from $0.3-1.2 \mathrm{~mm}$ in increments of $0.3 \mathrm{~mm}$. Then the conductor thickness that resulted in the most efficient actuator operation from the previous step was kept constant, and the conductor height was varied from 3$12 \mathrm{~mm}$ in $3 \mathrm{~mm}$ increments. Inter-turn gap was kept constant at $0.3 \mathrm{~mm}$ during the simulations. Eventually, the cross section dimensions that resulted in the most efficient switch operation were considered as the best parameter combination for the design space analyzed. To match the armature geometry used during latch design, both coils' external radii are assumed to be $5 \mathrm{~cm}$, while the internal radii for the top and bottom coil are $15 \mathrm{~mm}$ and $5 \mathrm{~mm}$, respectively. These radii were kept constants during all the simulations.

Figs. 8-9 show the simulation results obtained for different conductor cross-section dimensions. The tracking point used for armature and velocity calculation is circled in red in Fig. 7. As can be seen in Fig. 8, for a fixed $6 \mathrm{~mm}$ cross-section height, maximum armature velocity and displacement are obtained with a conductor thickness of $0.3 \mathrm{~mm}$. While for such conductor thickness, no significant improvement in velocity and displacement is noted beyond a $6 \mathrm{~mm}$ conductor crosssection height, Fig. 9. Thus a conductor cross-section of $0.3 \mathrm{~mm}$ by $6 \mathrm{~mm}$ is selected as the most advantageous for the armature geometry considered here. As can be seen in Fig. 8 the thicker the conductor (and therefore the lower the number of coil turns for the assumed constant coil inner and outer radii dimensions) the higher the amplitude of the observed speed oscillations. As discussed in [39], these speed oscillations result from the excitation of the armature natural vibration modes, which appear as armature bending. It must be noted that the larger the speed oscillations, the higher the energy dissipated as vibration. Thus although the amount of energy dissipated as vibration may be small, it is not surprising that for the cases analyzed here, the velocity trace that exhibits the smallest oscillations also corresponds to the case that achieves the highest speed/efficiency.

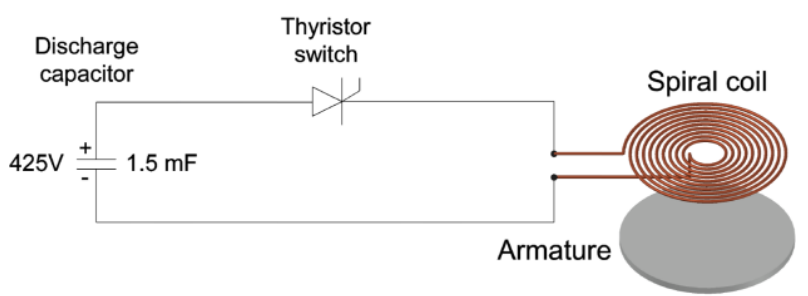

Fig. 6. Schematic diagram of the actuator electric circuit and FEA interface.

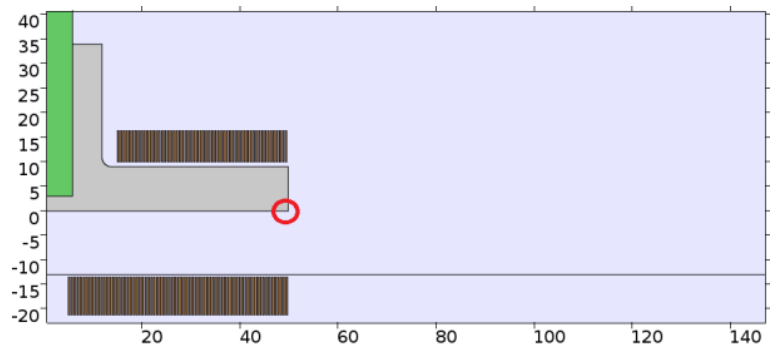

Fig. 7. Actuator FEA geometry.

TABLE I

VIPARAMETERS

\begin{tabular}{|l|l|}
\hline Parameter & Value \\
\hline Rated Voltage & $27 \mathrm{kV}$ \\
\hline Impulse withstand voltage & $100 \mathrm{kV}$ \\
\hline Rated current & $200 \mathrm{~A} \mathrm{RMS}$ \\
\hline Short circuit current $(0.5 \mathrm{~s})$ & $6 \mathrm{kA} \mathrm{RMS}$ \\
\hline Moving electrode mass & $227 \mathrm{~g}$ \\
\hline Stroke & $11.68 \mathrm{~mm}$ \\
\hline Max. allowable rebound & $25 \%$ of stroke \\
\hline Max. contact bounce duration & $2 \mathrm{~ms}$ \\
\hline Added contact force for rated short circuit & $330 \mathrm{~N}$ \\
\hline Mechanical life & 50,000 operations \\
\hline
\end{tabular}



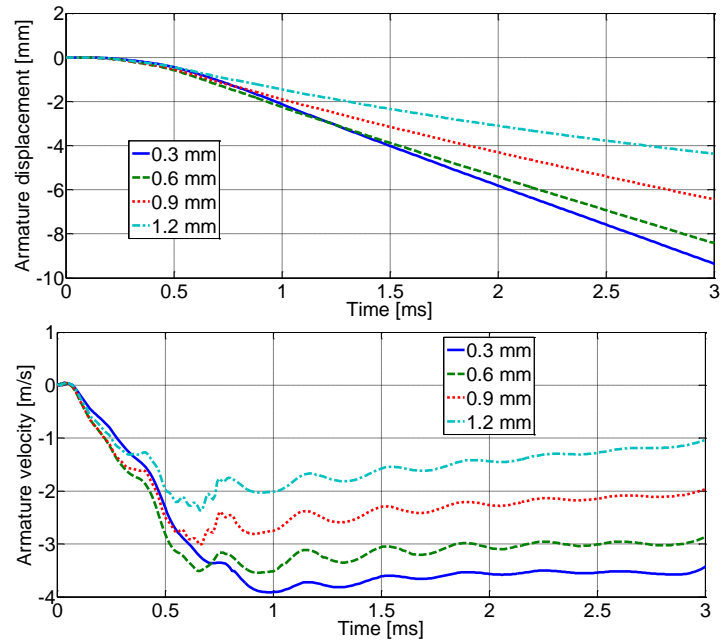

Fig. 8. FE simulations results for armature displacement (top) and velocity (bottom) for various conductor thicknesses and a $6 \mathrm{~mm}$ height.
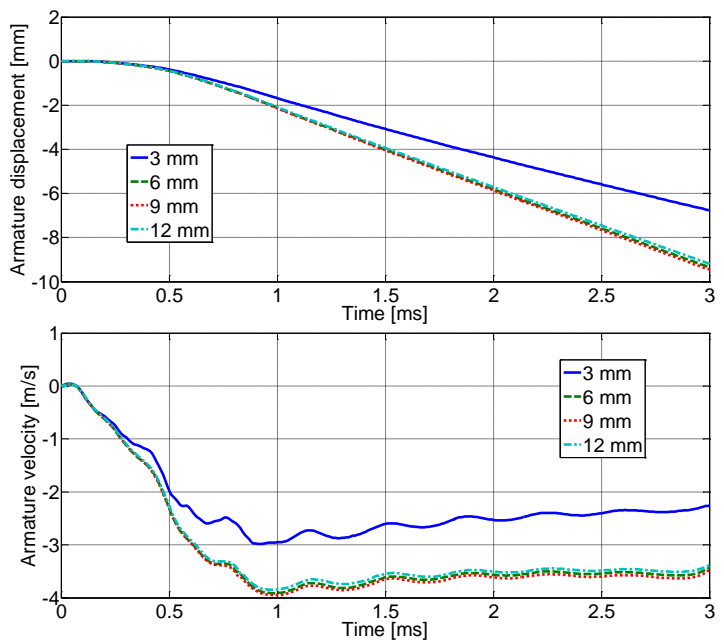

Fig. 9. FE simulation results for armature displacement (top) and velocity (bottom) for various conductor heights and a $0.3 \mathrm{~mm}$ thickness.

\section{ACTUATOR CONSTRUCTION AND TEST}

A test-rig based on the design described above was constructed to assess its performance under real conditions. $1 \mathrm{~mm}$ thick, 10mm wide CK75 +QT spring steel strip with yield strength of $\sim 1650 \mathrm{MPa}$ was used to build the latching system. To manufacture the slots required to insert the spring ribbons into the armature body (Fig. 10), Electrical Discharge Machining (EDM) was used. The use of the EDM technique avoids the application of any mechanical stress to the armature during the process, minimizing the possibility of inflicting any physical damage to the armature that may otherwise compromise its structural integrity. Lateral screws were added to the clamping structure of the spring ribbons to enable careful adjustment of the strips' strain. An extra pair of spring ribbons, with similar characteristics to those described above, was also added to provide the contact force required by the VI and to account for material uncertainties and geometric imperfections that may result in a lower force than that predicted by the FE simulations. The presence of the extra set of ribbons also helps to restrict unwanted armature movement, limiting any lateral displacement or unforeseen twist that could otherwise damage the VI bellows during operation.

According to the FE results in Fig. 5, a compressive strain of about $0.50 / 85$ is sufficient to allow adequate operation of the latching system over the VI stroke length. For such strain, a maximum ribbon stress of around $1400 \mathrm{MPa}$ is predicted by FE simulations, Fig. 11. The predicted ribbon stress is below the yield strength of the material employed, thus by using this strain, plastic deformation of the ribbons can be avoided. In contrast, although a 1/85 strain enables a wider operating range for the latching system (see Fig. 5) the material yield strength is exceeded, and permanent deformation of the spring ribbons may occur. In general, the lower the amount of repetitive mechanical stress applied to a mechanism, the higher the number of cycles to failure for a given material (s-n curve). Thus durability of the latching system is improved by minimizing the ribbon strain. Fig. 12 shows a picture of the armature-latching assembly.

The mass of the armature and latching ribbons was measured as $213 \mathrm{~g}$ and $30 \mathrm{~g}$, respectively. To verify that the latching system provides at least the $330 \mathrm{~N}$ of contact force recommended by the VI manufacturer a static load test of the latching mechanism was conducted. Due to the unusual dimensions of the spiral coil obtained from the FE simulations, no commercial conductor was suitable for coil manufacturing. Thus the coil conductor was hand built using $50 \mathrm{~mm}$ wide, $0.3 \mathrm{~mm}$ thick $\mathrm{C} 106$ copper strips. The copper strip was cut to the desired width and insulated manually using Kapton tape. Owing to the nature of the insulating process a relatively thick, $0.3 \mathrm{~mm}$, insulation layer resulted. For mechanical protection and to minimize bending during operation, the coils were inserted into $25 \mathrm{~mm}$ thick Tufnol sheets and encapsulated using epoxy resin. Fig. 13 shows one of the manufactured coils. In line with the FE design, asymmetric coils were utilized in the prototype. The coil parameters are listed in Table II for completeness. It should be noted that due to the coils' manufacturing process, small variations in its height were found, thus the table lists the coil's average conductor width.

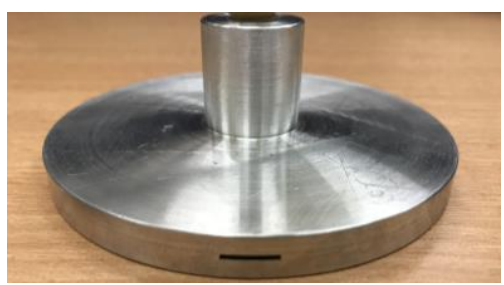

Fig. 10. 6082-T6 aluminum armature, slot detail.

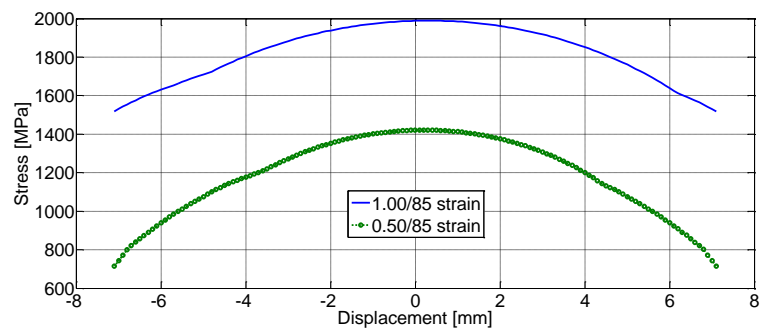

Fig. 11. Latching ribbon maximum stress obtained from FE simulation for two different compressive strains. 
TABLE II

COIL PARAMETERS

\begin{tabular}{|l|l|l|}
\hline Parameter & Top coil & Bottom coil \\
\hline Turns number & 59 & 65 \\
\hline Inner radius & $15 \mathrm{~mm}$ & $9.5 \mathrm{~mm}$ \\
\hline Outer radius & $50 \mathrm{~mm}$ & $50 \mathrm{~mm}$ \\
\hline Conductor width & $6.4 \mathrm{~mm}$ & $7.7 \mathrm{~mm}$ \\
\hline Conductor thickness & $0.30 \mathrm{~mm}$ & $0.30 \mathrm{~mm}$ \\
\hline
\end{tabular}

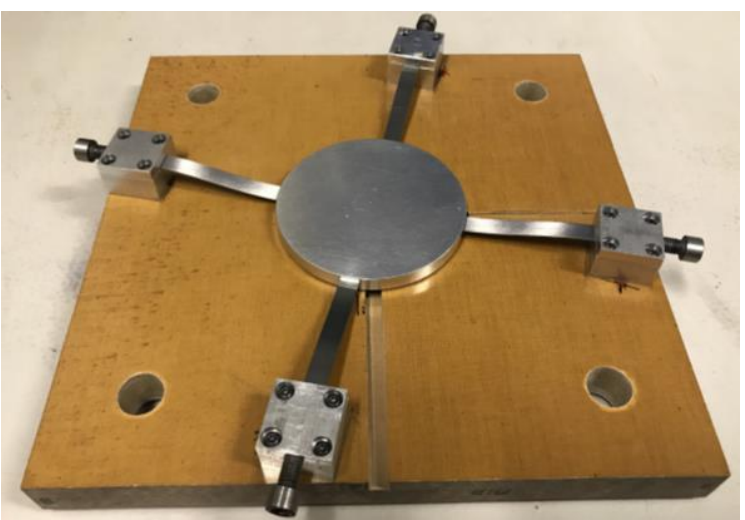

Fig. 12. Armature and latching system assembly.
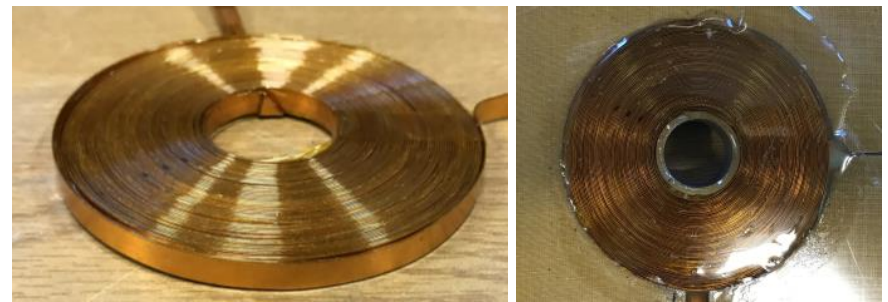

Fig. 13. Insulated spiral coil (left) and epoxy encapsulation (right).

For the assembly of the supporting structure, fiber glass reinforced plastic threaded WKT 472 M20 rods were employed. An M12 threaded rod of the same material was used as insulation/transmission rod. The use of threaded FRP material ensured excellent electrical insulation and magnetic neutrality, thus avoiding any interference on the coil-armature magnetic field interaction unaccounted in the simulations, with the added benefit of allowing fine adjustment of the necessary clearances and leveling of the horizontal surfaces. For testing purposes, the actuator-VI assembly was mounted on a fiber glass structure bolted to a heavy steel platform. The steel structure was firmly attached to the floor to avoid any unwanted displacement during the tests. Fig. 14 shows a picture of the assembled apparatus.

Coil current and capacitor voltage signals were recorded during the experiments using a CWT Rogowski coil and differential voltage probes, respectively. For displacement measurement a high speed Photron Fastcam SA-X2 color camera, recording at 20,000 fps and triggered by a TTL signal was employed. Displacement was obtained by video processing using an automatic video tracking application and velocity was calculated by numerical differentiation of the displacement signal. By directly deriving from the coil control circuit a TTL compatible signal, the start of the video recording and actuator operation were synchronized, thus mechanical displacement and electrical signals were acquired simultaneously.

To enable the use of the closing coil for active damping, a timing circuit with user selectable delay was included as part of the triggering circuit. For electric energy storage, one nonpolarized $1.3 \mathrm{kV} \mathrm{DC}, 1500 \mu \mathrm{F}, 6 \mathrm{kA}$ peak current NWL pulse capacitor was used to excite each coil independently. High power SEMIKRON SKKT 162/12E Thyristors were used to control the capacitor's discharge. Fig. 15 shows a schematic representation of the coil excitation circuit. Fig. 16 shows a picture of the setup used during the laboratory tests.

\section{A. Experimental results}

The total moving mass of the actuator-VI pair (501g including latching system), was calculated by adding each of the moveable components' mass. However in practice only a portion of the spring ribbons' mass is effectively displaced during operation, thus the contribution of the latching system to the total moveable mass is actually negligible. Fig. 17 compares FE predictions with electrical and mechanical quantities recorded during actuator operation with no damping and a capacitor initial voltage of $425 \mathrm{~V}$. Good agreement between numerical prediction and laboratory measurements is observed in the figure, thus validating the FE implementation.

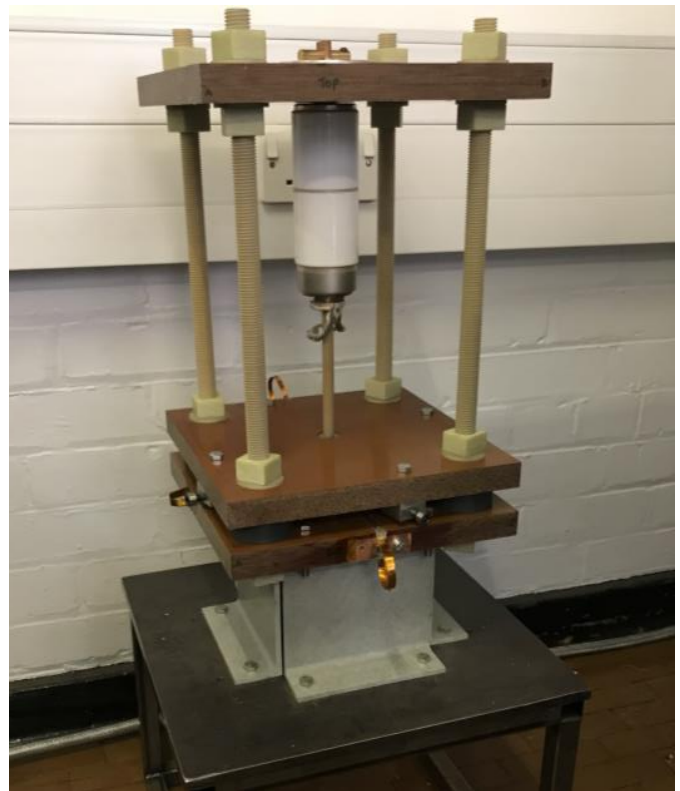

Fig. 14. TC actuator-VI assembly.

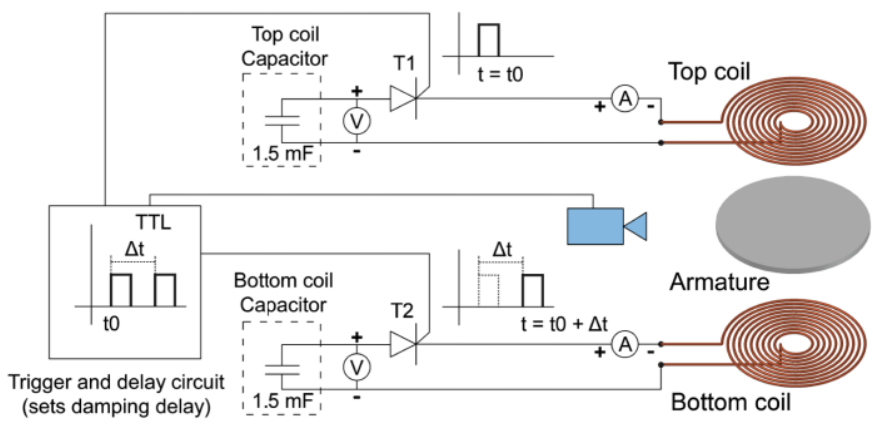

Fig. 15. Schematic diagram of the TC actuator experimental setup. 


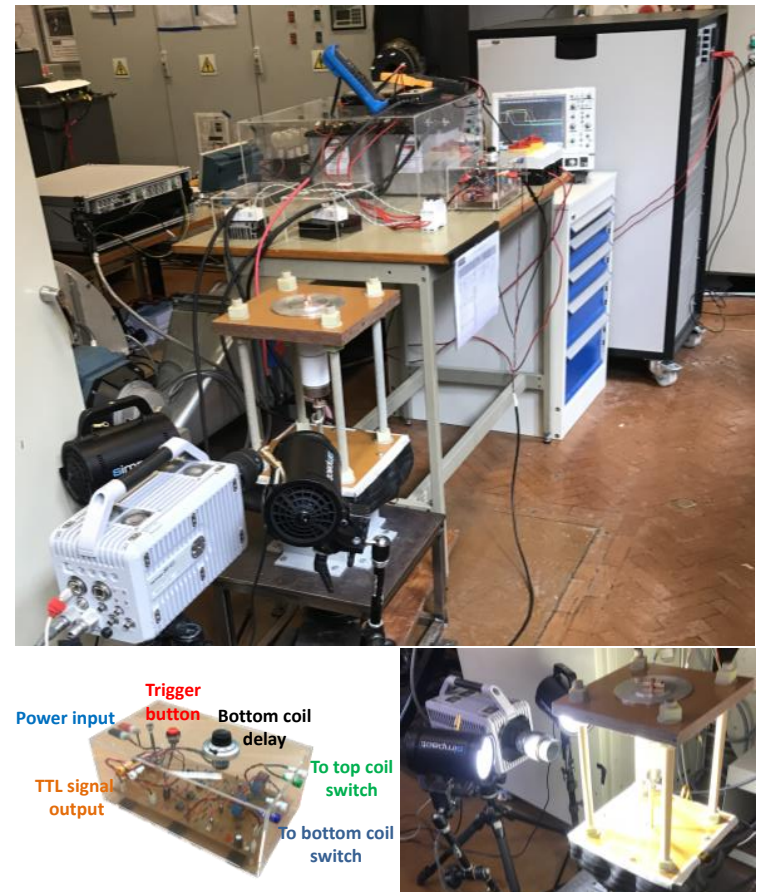

Fig. 16. Laboratory test setup (top), control box (bottom left), video recording detail (bottom right).

The electrical energy consumed during the test was calculated from the capacitor voltage trace as 122J. As can be observed in Fig. 17, the latching system is able to maintain the VI in the open position, even with no damping. Electrode bouncing of about $10 \%$ of the stroke length with a relatively long bouncing time, exceeding the switch opening time, is observed in the figure. To minimize the risk of arc restrike, excessive electrode bouncing must be avoided, thus damping must be added to reduce such a risk. It should be noted however that in a hybrid DC breaker, the transient voltage is usually clamped to about 1.5 times system voltage using suitably rated MOVs connected in parallel to the switch, $[10,12]$. Therefore an electrode gap significantly shorter than the VI nominal stroke is sufficient to avoid arc restrike in such applications [41]. For instance, for the VI used in the experiments, a $6 \mathrm{~mm}$ electrode gap would suffice to provide the required voltage isolation for the assumed 1.5 times rated voltage. A $6 \mathrm{~mm}$ displacement is achieved in less than $2 \mathrm{~ms}$ for the proposed design, which is compatible with the operation times demanded from DC breakers. Thus for hybrid DC breaker applications the electrode bouncing observed in the experiments is acceptable. Nevertheless the effects of active damping on the actuator operation and the energy required for its implementation are assessed below for the sake of completeness.

Fig. 18 shows the armature displacement for different capacitor voltages applied to the closing coil when active damping is enabled $3 \mathrm{~ms}$ after the "open" command is issued. As can be seen in Fig. 18, bouncing is practically eliminated with a $335 \mathrm{~V}$ damping voltage. The effect of damping delay for such a voltage is examined in more detail in Fig. 19. In Fig. 19 , maximum displacement with minimum bouncing is achieved with $335 \mathrm{~V}$ damping and $3.6 \mathrm{~ms}$ delay. This is further illustrated in Fig. 20, where electrode and armature velocity are shown for this condition.


Fig. 17. Simulation vs measurement comparison for capacitor voltage (top), coil current (middle) and electrode displacement/velocity (bottom) for actuator operation with no damping.

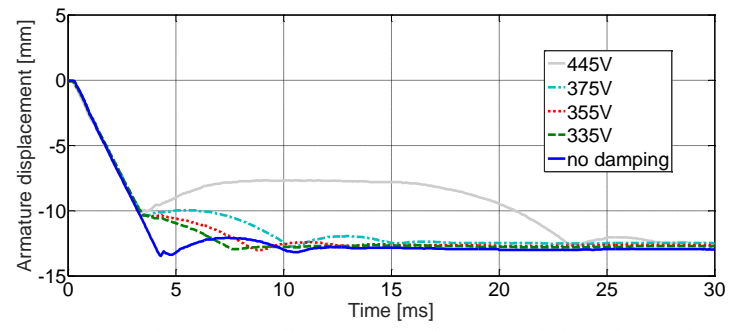

Fig. 18. Measured armature displacement for several damping voltages.

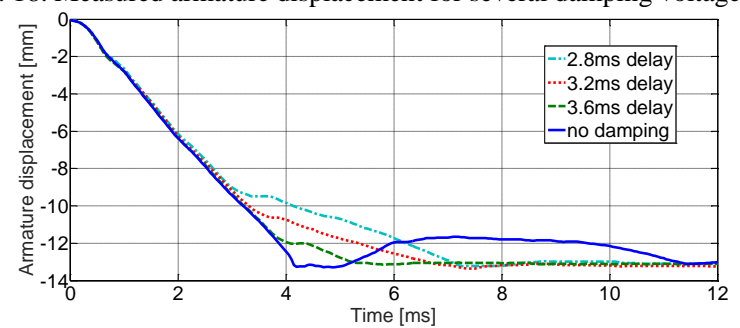

Fig. 19. Measured armature displacement for several damping delays.

From the camera's point of view, the electrode velocity obtained under such operating conditions shows only a small magnitude ripple after damping is applied, while the armature speed oscillations are more pronounced, exaggerated by the relatively large deflection that the armature suffers near its external edges [39]. Fig. 21 shows the top and bottom coil current and capacitor voltage traces recorded for the same scenario. From these results the energy used for armature acceleration and active damping is calculated as $124 \mathrm{~J}$ and $59 \mathrm{~J}$, respectively. The results in Figs. 19-21 show that active damping may be effectively implemented using only $47 \%$ of the energy required for armature acceleration. Thus with careful actuator design, the application of active damping is not necessarily a costly solution in terms of energy usage.

Since the main goal during the experiments was to test the efficacy of the proposed latching system and validate the 
theoretical performance brought by the high density coils, a conservative armature thickness (with a safety factor of 4) was chosen for the experiments. From the simulations the maximum stress that the $9 \mathrm{~mm}$ armature experiences during operation was calculated as $60 \mathrm{MPa}$, which is about a quarter of the 6082-T6 aluminum yield strength $(260 \mathrm{MPa})$. Thus further gains in the actuator performance may be possible by, for example, optimizing armature size. This possibility is explored below.

By reducing armature thickness, while still operating within the material mechanical limits, the system moveable mass can be reduced. Fig. 22 shows armature displacement for different armature thicknesses and a $425 \mathrm{~V}$ excitation; the armatures masses are included in the figure for reference. As can be seen in the figure, the thinnest, $4 \mathrm{~mm}$ armature (which according to the simulations is subject to a maximum stress of $248 \mathrm{MPa}$ ) exhibits a drop in performance compared with the $9 \mathrm{~mm}$ armature. In contrast, the $6 \mathrm{~mm}$ thick armature (which is subject to a maximum stress of $128 \mathrm{MPa}$ ) shows a small improvement compared to the $9 \mathrm{~mm}$ case. Thus a reduction in armature mass can improve actuator performance, but not in all cases. Armature mass must not be assumed as "dead weight", armature volume and geometry play active roles in the actuator performance. Coil-armature magnetic field interaction has a bearing on system performance [19, 21]. For instance, if the armature is too thick, there will be armature material which has zero current density due to the skin effect, which pushes the induced disc current towards the outer surface. This material is not active electromagnetically and will simply add mass and therefore reduce the acceleration. In contrast, if the disc is thin and comparable to the skin depth, this will interfere with the induced current distribution, producing additional resistive losses and reducing the repulsive force, which also reduces the actuator performance. Thus, as is shown by the simulation results in Fig. 22, the most convenient armature thickness is not necessarily the lowest thickness able to withstand the system mechanical stresses. The optimal disc thickness for actuator performance is a delicate balance between the electrical response and the mechanical integrity to meet the actuator requirements.

For the considered design, actuator performance can be further improved by increasing coil conductor density by, for example, improving coil manufacturing and reducing conductor insulation thickness; thus enabling the construction of a coil with a higher number of turns with identical footprint. This possibility is corroborated by the results in Fig. 23, where FE simulation results for armature displacement and velocity show increments, as the coil inter-turn gap decreases and coil turn number rises. As discussed before, the armature's reaction time tends to grow as the coil turn number (inductance) increases. However for the conductor crosssection considered in this work, as the turn number grows the average speed within the $2 \mathrm{~ms}$ operation period also grows, thus the time to reach the necessary $6 \mathrm{~mm}$ electrode gap drops with the increase in conductor density. However if the coil turn number is augmented excessively, actuator performance may degrade, since the current's peak value and rate of change (di/dt) would be severely limited by the coil's resistance and inductance, respectively [19, 21]. For instance, although at the end of the $3 \mathrm{~ms}$ period shown in Fig. 23 the longer displacement is achieved with the 98 turn coil, the necessary $6 \mathrm{~mm}$ electrode gap is reached first with the 87 turn coil. Thus for the intended application, the 87 turn coil is more convenient than the coil with the highest density. This example shows the key role that coil sizing plays in achieving the best performance for the intended application. Optimal coil turn number will vary from application to application, depending on all the system parameters [21, 42].

To assess the gains offered by the reduction on the moveable mass brought out by the implementation of the proposed latching system FE simulations were conducted. In the simulations different moveable masses were added to mimic the presence of alternative latching mechanisms. The best performing configuration that uses the $6 \mathrm{~mm}$ thick armature, the 87 turn top coil and $425 \mathrm{~V}$ excitation is considered in the analysis. It should be noted that in practice the excess mass will depend entirely on the implementation of the alternative latching system, therefore establishing a precise additional mass value is unrealistic. Thus a relatively wide excess mass range was used in the simulations to try to include all the different alternatives. The calculated travel time required to reach the necessary $6 \mathrm{~mm}$ electrode gap for different excess masses is shown in Fig. 24.

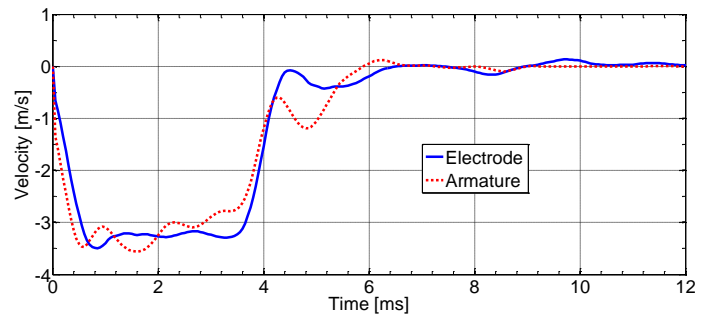

Fig. 20. Electrode and armature velocities calculated from experimental measurements with $335 \mathrm{~V}$ active damping enabled at $3.6 \mathrm{~ms}$.
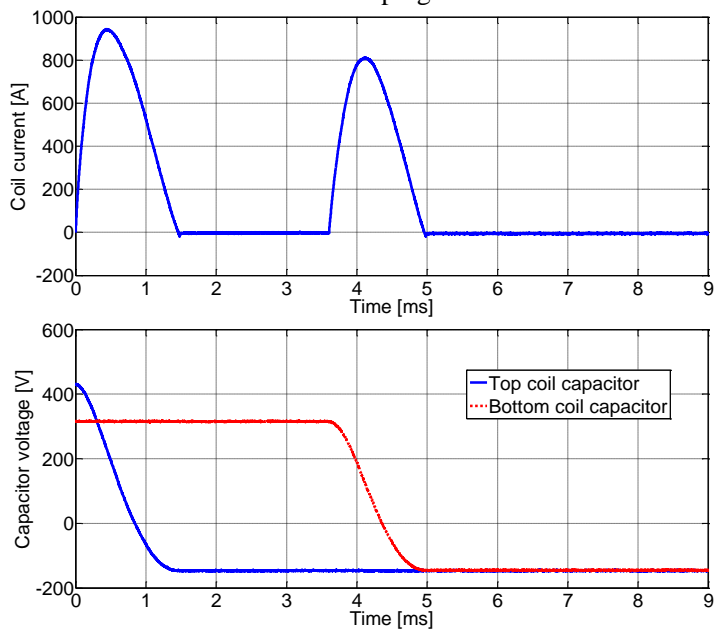

Fig. 21. Measured coil currents (top) and capacitor voltage (bottom) for $335 \mathrm{~V}$ active damping enabled at $3.6 \mathrm{~ms}$.

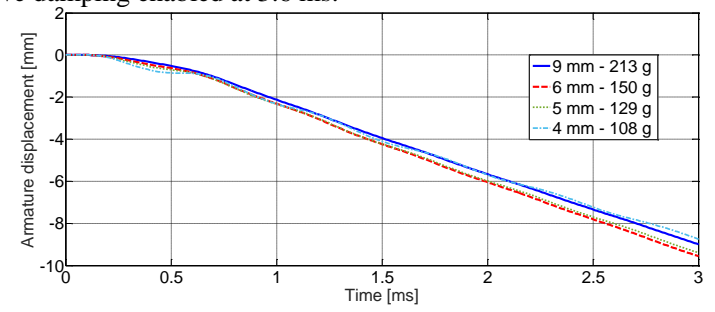

Fig. 22. FE simulation results for armature displacement for different armature thicknesses and $425 \mathrm{~V}$ excitation. 

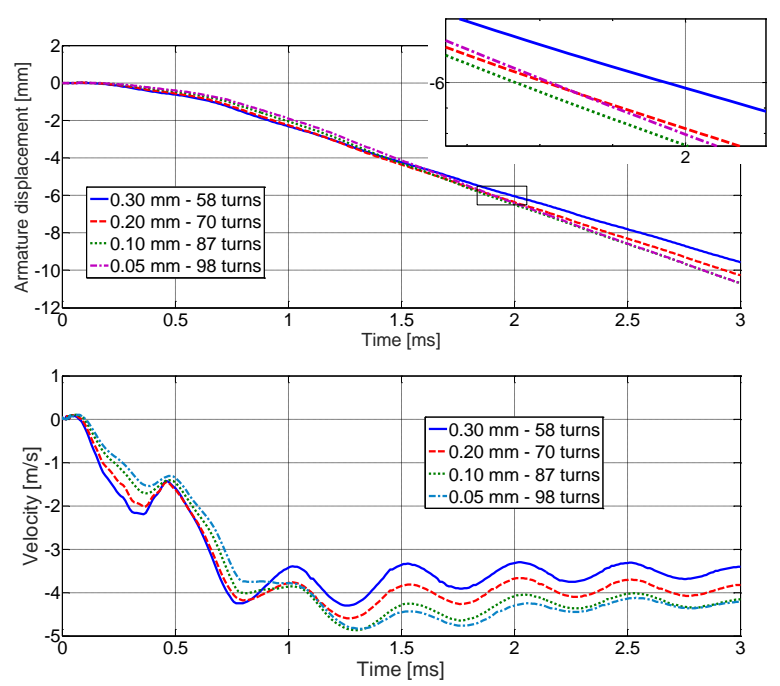

Fig. 23. FE simulation results for armature displacement and velocity for different coil conductor densities with a $425 \mathrm{~V}$ excitation and a $6 \mathrm{~mm}$ armature.

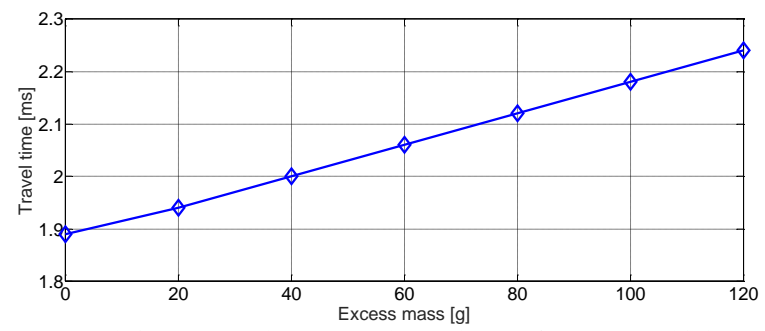

Fig. 24. Travel time to reach a $6 \mathrm{~mm}$ electrode gap for a $6 \mathrm{~mm}$ thick armature with an 87 turn top coil and $425 \mathrm{~V}$ excitation for moving masses in excess of $440 \mathrm{~g}$.

As can be seen in Fig. 24 the actuator performance decreases even for very small excess mass values. In DC protection, where short circuit currents with very high di/dt are common, even fractions of milliseconds may represent an increase of several hundreds of amps in fault current. Thus any gain in breaker performance, obtained without any sacrifice, is welcome. Thus in addition to allowing a simplified design, quantifiable gains in actuator performance must be expected from the implementation of the proposed latching system. It is important to mention that during the laboratory tests, hundreds of open/close operations of the prototype, with and without damping, were conducted with no sign of degradation on the latching system or on any of the other actuator-switch pair components, thus highlighting the robustness of the proposed design. However some degradation may be expected as the VI and spring ribbons approach its mechanical life limits. Thus thousands of operations are needed to ensure the suitability of the design for mission critical applications in DC protection.

\section{CONCLUSIONS}

In this paper a light, compact, low energy TC actuator design for the operation of a MV vacuum CB is proposed. High density coils, with a large height-to-width conductor cross section ratio are used to operate the VI in times suitable for MVDC protection. The use of a buckling based latching mechanism, directly attached to the armature body, is proposed to minimize actuator moving mass. Simulation results are used to show that the operating range of the proposed latching systems is dictated by the strain in the spring ribbons. By the careful selection and adjustment of the spring ribbon strain, the latching system is made to operate within the material mechanical limits. Effectiveness of the latching design is demonstrated by simulations and experimental results which show adequate contact and latching forces for the operation of a $27 \mathrm{kV}$ VI. It is shown that, even with no damping, the proposed design is suitable for DC applications. It is demonstrated that with adequate sizing of the actuator coils, effective active damping is realizable using a fraction of the energy required for switch opening. FE simulations are used to demonstrate that for the proposed design, actuator performance may be further improved by increasing the coil packing factor by reducing the conductor insulation thickness. However limits exist, as it is shown that an excessive number of coil turns may result in an armature reaction delay that may be detrimental for the intended actuator application. Coil and conductor cross-section geometries are critical for efficient operation of the TC actuator. It is also shown that any excess mass applied to the proposed design results in a quantifiable increase in travel time. Thus in addition to enabling a simplified actuator design, the reduction in moving mass allowed by the implementation of the proposed latching systems should bring performance advantages compared to actuators equipped with alternative latching mechanisms.

The integral design of coil, armature and latching system are necessary to achieve the highest actuator performance with reduced energy usage.

\section{REFERENCES}

[1] "Next generation integrated power system: NGIPS technology development roadmap," Naval Sea Systems Command, Nov. 2007.

[2] N. Doerry and J. Amy Jr., "The road to MVDC," ASNE Intelligent Ships Symp., Philadelphia PA, 2015.

[3] N. Doerry and J. Amy Jr., "DC voltage interface standards for naval applications," IEEE Electric Ship Technologies Symp., Old Town Alexandria, VA, Jun. 2015.

[4] P. Gemin et al., "Architecture, voltage and components for a turboelectric Distributed propulsion electric grid (AVC-TeDP)," NASA/CR-2015-218713, 2015.

[5] F. Mura, and Rik. W. De Doncker, "Design aspects of a mediumvoltage direct current (MVDC) grid for a university campus," 8th Int. Conf. on Power Electronics - ECCE Asia, 2011.

[6] F. Mura, and Rik. W. De Doncker, "Preparation of a mediumvoltage DC grid demonstration project," E.ON Energy Research Center Series, vol. 4, issue 1, pp. 1-32.

[7] "MVDC technology study - market opportunities and economic impact", TNEI, Feb. 2015.

[8] CIGRE WG B4.60, "Designing HVDC grids for optimal reliability and availability performance", Technical brochure, December 2017.

[9] C. Peng, I. Husain, A. Q. Huang, B. Lequesne and R. Briggs, "A Fast Mechanical Switch for Medium-Voltage Hybrid DC and AC Circuit Breakers," in IEEE Transactions on Industry Applications, vol. 52, no. 4, pp. 2911-2918, July-Aug. 2016.

[10] J. Häfner, B. Jacobson, "Proactive Hybrid HVDC Breakers - A key innovation for reliable HVDC grids," (Cigré Bologna, Paper 0264, 2011)

[11] X. Pei, O. Cwikowski, A. C. Smith and M. Barnes, "Design and Experimental Tests of a Superconducting Hybrid DC Circuit Breaker," in IEEE Transactions on Applied Superconductivity, vol. 28, no. 3, pp. 1-5, April 2018.

[12] L. Ängquist, S. Norrga and T. Modéer, "A new dc breaker with reduced need for semiconductors," 2016 18th European Conference on Power Electronics and Applications (EPE'16 ECCE Europe), Karlsruhe, 2016, pp. 1-9. 
[13] L. Zhang, R. Woodley, X. Song, S. Sen, X. Zhao and A. Q. Huang, "High current medium voltage solid state circuit breaker using paralleled 15kV SiC ETO," 2018 IEEE Applied Power Electronics Conference and Exposition (APEC), San Antonio, TX, 2018, pp. 1706-1709.

[14] T. Erikksson, M. Backman, S. Halen, "A low loss mechanical HVDC breaker for HVDC Grid applications", CIGRE 2014.

[15] W. Holaus, K. Frohlich, "Ultra-fast switches- a new element for medium voltage fault current limiting switchgear," Power Engineering Society Winter Meeting, 2002. IEEE, vol.1, no., pp.299,304 vol.1, 2002

[16] W. Wen et al., "Research on Operating Mechanism for Ultra-Fast 40.5-kV Vacuum Switches," in IEEE Transactions on Power Delivery, vol. 30, no. 6, pp. 2553-2560, Dec. 2015.

[17] M. Steurer, K. Frohlich, W. Holaus, and K. Kaltenegger, "A novel hybrid current-limiting circuit breaker for medium voltage: Principle and test results," IEEE Trans. Power Del., vol. 18, no. 2, pp. 460-467, Apr. 2003.

[18] J. Magnusson, O. Hammar, G. Engdahl "Modelling and Experimental Assessment of Thomson Coil Actuator System for Ultra Fast Mechanical Switches for Commutation of Load Currents," International Conference on New Actuators and Drive Systems, Bremen, 14-16 Jun 2010.

[19] A. Bissal, J. Magnusson, G. Engdahl, "Electric to Mechanical Energy Conversion of Linear Ultrafast Electromechanical Actuators Based on Stroke Requirements," Industry Applications, IEEE Transactions on, vol.51, no.4, pp.3059,3067, July-Aug. 2015.

[20] A. Bissal, J. Magnusson, G. Engdahl, "Comparison of Two UltraFast Actuator Concepts," Magnetics, IEEE Transactions on, vol.48, no.11, pp.3315,3318, Nov. 2012

[21] D.S. Vilchis-Rodriguez, R. Shuttleworth, M. Barnes, "Finite element analysis and efficiency improvement of the Thomson coil actuator", 8th IET International Conference on Power Electronics, Machines and Drives (PEMD 2016), Glasgow, UK, 19-21 April 2016.

[22] A. Bissal, E. Salinas, G. Engdahl, and M. Ohrstrom, "Simulation and verification of Thomson actuator systems," in Proc. COMSOL Conf., Paris, France, 2010, pp. 1-6.

[23] C. Peng, I. Husain, A. Q. Huang, B. Lequesne and R. Briggs, "A Fast Mechanical Switch for Medium-Voltage Hybrid DC and AC Circuit Breakers," in IEEE Transactions on Industry Applications, vol. 52, no. 4, pp. 2911-2918, July-Aug. 2016.

[24] E. Dong, P. Tian, Y. Wang, and W. Liu, "The design and experimental analysis of high-speed switch in $1.14 \mathrm{kV}$ level based on novel repulsion actuator," in Electric Utility Deregulation and Restructuring and Power Technologies (DRPT), 2011 4th International Conference on,pp.767-770, 6-9 July 2011.

[25] Z. Yuan, J. He, Y. Pan, X. Jing, C. Zhong, N. Zhang, X. Wei, and G. Tang, "Research on ultra-fast vacuum mechanical switch driven by repulsive force actuator," Rev. Sci. Instrum. Vol. 87, issue 12, 2016.

[26] C. Peng, X. Song, A. Q. Huang and I. Husain, "A Medium-Voltage Hybrid DC Circuit Breaker-Part II: Ultrafast Mechanical Switch," in IEEE Journal of Emerging and Selected Topics in Power Electronics, vol. 5, no. 1, pp. 289-296, March 2017.

[27] W. Wen et al., "Research on Operating Mechanism for Ultra-Fast 40.5-kV Vacuum Switches," in IEEE Transactions on Power Delivery, vol. 30, no. 6, pp. 2553-2560, Dec. 2015.

[28] Dong enyuan, Wang yongxing, Cong jiyuan and Zou jiyan, "The analysis of high-speed repulsion actuator and performance comparisons with permanent magnetic actuator in vacuum circuit breaker," 2008 23rd International Symposium on Discharges and Electrical Insulation in Vacuum, Bucharest, 2008, pp. 189-191.

[29] C. Peng et al., "Active Damping of Ultrafast Mechanical Switches for Hybrid AC and DC Circuit Breakers," in IEEE Transactions on Industry Applications, vol. 53, no. 6, pp. 5354-5364, Nov.-Dec. 2017.

[30] E. Dong, P. Tian, Y. Wang and W. Liu, "The design and experimental analysis of high-speed switch in $1.14 \mathrm{kV}$ level based on novel repulsion actuator," 2011 4th International Conference on Electric Utility Deregulation and Restructuring and Power Technologies (DRPT), Weihai, Shandong, 2011, pp. 767-770.

[31] D.S. Vilchis-Rodriguez, R. Shuttleworth, A.C. Smith, M. Barnes, "A comparison of damping techniques for the soft-stop of ultra-fast linear actuators for HVDC breaker applications", The 9th
International Conference on Power Electronics, Machines and Drives (PEMD), Liverpool, UK, 2018, pp. 1-6.

[32] A. Bissal, E. Salinas, J. Magnusson and G. Engdahl, "On the Design of a Linear Composite Magnetic Damper," in IEEE Transactions on Magnetics, vol. 51, no. 11, pp. 1-5, Nov. 2015, Art no. 8003305.

[33] Y. Wu et al., "A New Thomson Coil Actuator: Principle and Analysis," in IEEE Transactions on Components, Packaging and Manufacturing Technology, vol. 5, no. 11, pp. 1644-1655, Nov. 2015.

[34] Jin Qiu, J. H. Lang and A. H. Slocum, "A curved-beam bistable mechanism," in Journal of Microelectromechanical Systems, vol. 13, no. 2, pp. 137-146, April 2004.

[35] P. Cazottes A. Fernandes J. Pouget and M. Hafez "Bistable buckled beam: Modeling of actuating force and experimental validations " Journal of Mechanical Design vol. 131, no. 10, Octuber 2009.

[36] T. Abe, Y. Furuya and S. Matsuoka: 'Gigacycle fatigue properties of $1800 \mathrm{MPa}$ class spring steels', Fatigue Fract. Eng. Mater. Struct., vol. 27, no. 2, pp. 159-167. 2004.

[37] A. Tump, R. Brandt, "Graded high-strength spring-steels by a special inductive heat treatment", IOP Conference Series: Materials Science and Engineering, 2016.

[38] V.J. Matjeke, G. Mukwevho, A.M. Maleka, J.W. van der Merwe, "Effect of heat treatment on strength and ductility of $52 \mathrm{CrMoV} 4$ spring steel", IOP Conf. Series: Materials Science and Engineering 2018.

[39] D. S. Vilchis-Rodriguez, R. Shuttleworth and M. Barnes, "Modelling Thomson Coils With Axis-Symmetric Problems: Practical Accuracy Considerations," in IEEE Transactions on Energy Conversion, vol. 32, no. 2, pp. 629-639, June 2017.

[40] Jennings vacuum capacitors, relays, interrupters, contactors, DC contactors, https://new.abb.com/low-voltage/products/cableaccessories-and-apparatus/jennings-vacuum-capacitors-relaysinterrupters-contactors-dc-contactors, accessed January 16, 2019.

[41] L. Ängquist, A. Baudoin, S. Norrga, S. Nee and T. Modeer, "Lowcost ultra-fast DC circuit-breaker: Power electronics integrated with mechanical switchgear," 2018 IEEE International Conference on Industrial Technology (ICIT), Lyon, 2018, pp. 1708-1713.

[42] M. Pham, Z. Ren, W. Li and C. S. Koh, "Optimal Design of a Thomson-Coil Actuator Utilizing a Mixed-Integer-DiscreteContinuous Variables Global Optimization Algorithm," in IEEE Transactions on Magnetics, vol. 47, no. 10, pp. 4163-4166, Oct. 2011. 